\title{
The Introduction and Application of a Selective Directional Capability of the Image Contrast Transfer Function in the ImageJ 'SMARTeR” Package
}

\author{
B. J. Griffin ${ }^{1}$, D.C. Joy ${ }^{2}$ and J. R. Michael ${ }^{3}$
}

1 Centre for Microscopy, Characterization and Analysis, The University of Western Australia, Crawley, WA Australia 6009

2 Center for NanoPhase Materials Science, Oak Ridge National Laboratory, Oak Ridge, TN 37831

3 Sandia National Laboratories, PO Box 5800, Albuquerque, NM 87185-0886

The ImageJ based "SMARTE" SEM performance and/or image characterization software provides a range of useful data, including resolution, signal-to-noise and contrast transfer function (CTF) data [1]. The existing package subroutine dealing with the CTF uses a full 360 degree radial integration of the Fast Fourier Transform (FFT) derived from the image to generate the CTF. This approach has been used successfully to characterize modern SEM [2].

To better understand the image data and CTF analysis it was suggested [3] that the capability to interactively select the angular range and direction of the FFT data for the CTF derivation would be useful. The specific code for the radial selection was sourced from the NIH ImageJ Library [4] and has been introduced into the CTF routine of the SMARTE program (now SMARTeR). A range of test images were created and to model a range of potential artifacts. These were analysed using the new selective SMARTeR CTF routine and the results demonstrated an improved sensitivity to artifact than seen in the full radial analysis.

Recent instrument characterization studies of high resolution SEM and also of the helium-based scanning ion microscope (SIM) have provided an example of unusual and inexplicable resolution results and CTF curves. Very low (and improbable) resolution data were measured, for example. Also and notably some CTF curves displayed a broad plateau in the finer spatial dimension although nothing was immediately evident in the source image. This type of CTF curve is clearly distinct from 'normal' curves (figure 1) [2] and also warranted further investigation.

Radial CTF analysis of the SEM images with 'anomalously' low resolution results have been performed. In one case, for example, automatically measured (SMARTeR routine) resolution figure of " $0.14 \mathrm{~nm}$ " is found to be a vibration artifact visible at $0.24 \mathrm{~nm}$ in the vertical analysis of the CTF, with real image resolution around $0.5 \mathrm{~nm}$ identifiable in the horizontal CTF data (figure 2).

The reprocessing of the "plateau CTF" image data by selecting a 90 degree integration range in both the horizontal and vertical directions clearly distinguishes this anomaly into an artifact associated with the horizontal dimension of the image. The original image was collected using the common 'line-average' method and this was found to be the key. Modelling of horizontal sample drift, by averaging a series of the same image with incremental horizontal offsets to give a single pixel total "drift" generated a similar plateau effect in the horizontal CTF. Re-collection of a second image from the sample without 'line averaging' eliminated the effect (figure 3). Testing of the line integration mode on a matching (same manufacturer) SEM did not show the same effect however it was found to be present on a second helium-based SIM. One possibility for this effect is that the reproducibility of the positioning of the helium beam during line integration was not ideal.

The directional analysis of the CTF has also been found to allow identification of variations in $\mathrm{S} / \mathrm{N}$ and vibration effects in characterization imaging and is suggested to be an additional, useful tool for performance characterization of scanning ion and electron microscopes. 
[1] Joy, D.C. et al. (2007), Microsc. Microanal., 13 (S2:Proc.) 1682CD

[2] Michael, J.R. et al. (2009) Microsc Microanal, 15 (S2:Proc.), 660CD

[3] He SIM access was generously provided through the ALIS Group of Carl Zeiss SMT. John Notte and Ray Hill, amongst many others of that group, provided excellent insight and comment including suggestions of performing radial CTF analysis.

[4] IMAGEJ written by Wayne Rasband is available from (http://rsb.ifo.nih.gov/ij/), SMARTeR plug-in is available from B.J. Griffin (brendan.griffin@uwa.edu.au) or D. C. Joy (djoy@utk.edu ).
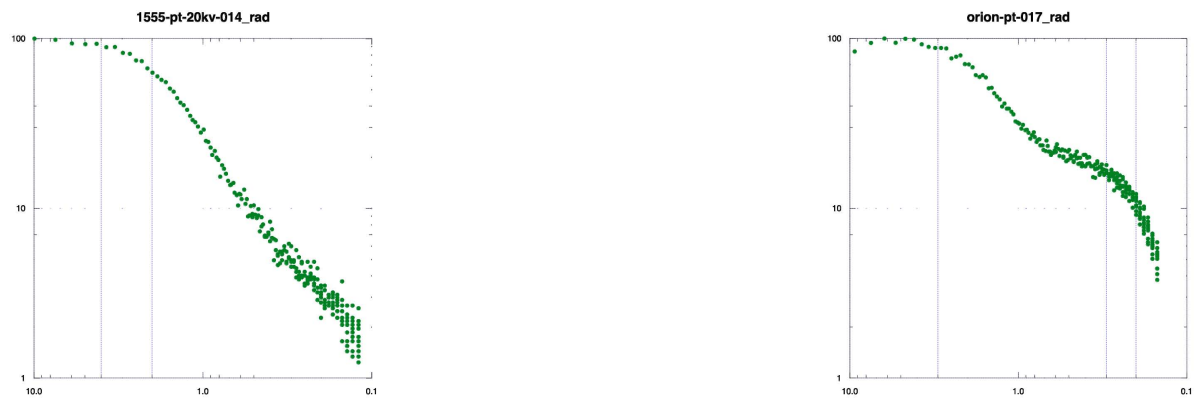

Figure 1: examples of the "normal" and "plateau" CTF results from scanned electron $(l)$ and ion $(r)$ microscopes.

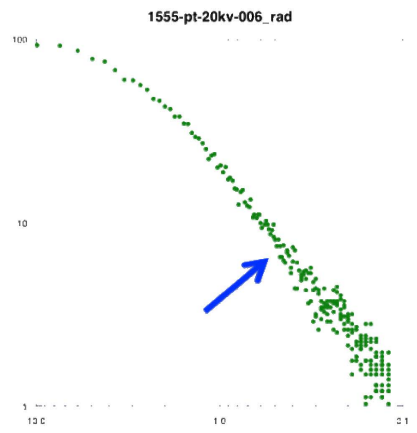

Figure 2(a): Full 360 degree CTF analysis with noise floor (resolution) around 0.5 $\mathrm{nm}$ in an SEM image

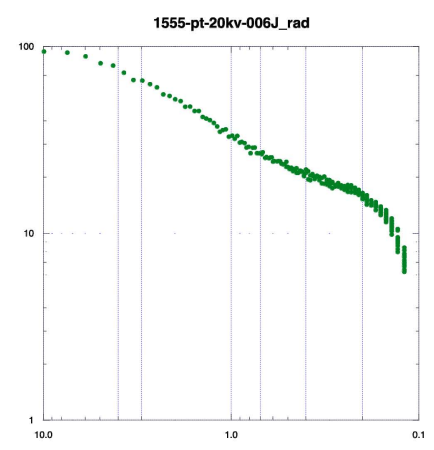

Figure 3(a): 1 pixel offset model image - full 360 degree CTF analysis

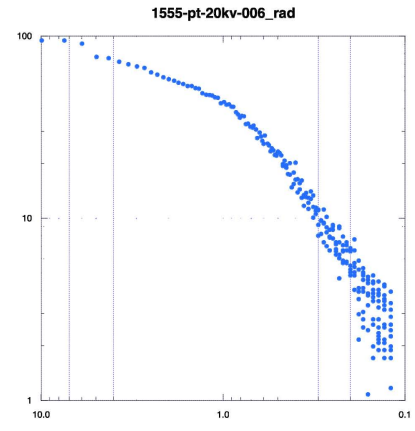

Figure 2(b): Horizontal CTF Figure 2(c): Vertical CTF analysis analysis that matches the full 360 result.

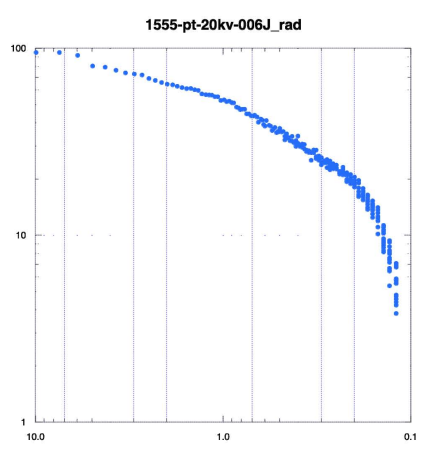

Figure 2(b): 1 pixel offset model image - horizontal CTF analysis showing a marked 'plateau' effect.

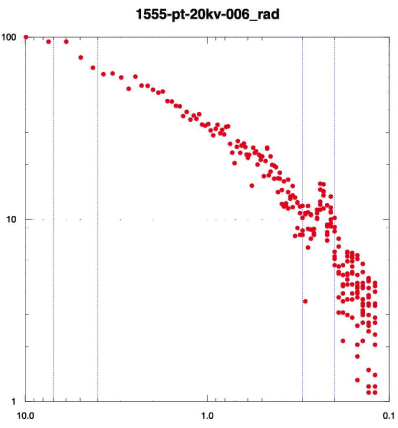

showing a ?vibration spike at 0.44 $\mathrm{nm}$ that matches the $360 \mathrm{deg}$. automatic resolution measurement.

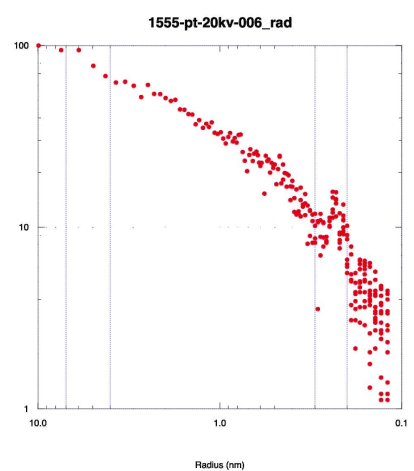

Figure 2(c): 1 pixel offset model image - vertical CTF analysis with vibration spike unchanged as per 2(c). 